\title{
Effects of avocado/soybean unsaponifiables (ASU) on the treatment of ligature-induced periodontitis in rats
}

\section{Guilherme José Pimentel Lopes de OLIVEIRA(a) Luiz Guilherme Freitas de PAULA ${ }^{(b)}$ João Antônio Chaves de SOUZA(c) Rubens SPIN-NETO(d) Andreas STAVROPOULOS(e) Rosemary Adriana Chiérici MARCANTONIO(a)}

(a) Universidade Estadual de São Paulo - UNESP, School of Dentistry of Araraquara, Department of Diagnosis and Surgery, Araraquara, SP, Brazil.

(b)Centro Universitário Unievangélica, Department of Periodontology, Anápolis, GO, Brazil.

(c) Universidade Federal de Goiás - UFG, Dental School, Department of Stomatological Science, Goiânia, GO, Brazil.

(d)Aarhus University, Department of Dentistry, Section of Oral Radiology, Aarhus, Denmark.

(e) Malmo University, Faculty of Odontology, Department of Periodontology and Community Dentistry, Malmo, Sweden.

Declaration of Interest: The authors certify that they have no commercial or associative interest that represents a conflict of interest in connection with the manuscript.

\section{Corresponding Author:}

Rosemary Adriana Chiérici Marcantonio

E-mail: adriana@foar.unesp.br

htrp://doi.org/10.1590/1807-3107BOR-2017.vol31.0028

Submitted: Sep 01, 2016

Accepted for publication: March 06, 2017

Last revision: March 13, 2017
Abstract: The purpose of this study is to evaluate the effect of the avocado/soybean unsaponifiables (ASU) on the treatment of induced periodontitis in rats. Periodontitis was induced in 84 rats via ligature placement around the second upper molar, which was removed after 7 days, and scaling and root planning (SRP) was performed at this time. Subsequently, the rats were randomly allocated to four groups with 21 animals each: OneSRP group in which saline solution was administered (SS), and three groups in which ASU was administered $(0.6 \mathrm{~g} / \mathrm{kg} /$ day), beginning either 7 days before the induction of periodontitis (SRP/ASU-7), on the day of periodontitis induction (SRP/ASU0), or on the day of treatment (SRP/ASU+7). ASU and SS were administered daily by gavage until the sacrifice of the animals $(7,15$, and 30 days after SRP). The \% bone in the furcation area was evaluated by histomorphometry and micro-CT. The expression of proteins (TRAP, RANKL, and alkaline phosphatase) and mRNA (IL-1 $\beta$, TNF-a, IL-6, RANKL, and alkaline phosphatase) were evaluated by immunohistochemistry and qPCR. The SRP/ASU+7 group presented a higher percentage of bone fill in the furcation area and higher expression of alkaline phosphatase than in the SRP group (at 7 and 30 days, respectively). The SRP/ASU0 and SRP/ASU+7 groups presented lower expression levels of RANKL mRNA than the SRP and SRP/ASU-7 groups at 15 days. ASU administration on the day of the SRP treatment of the ligature-induced periodontitis promoted subtle beneficial effects on periodontal repair following the treatment of induced periodontitis within the experimental period of 7 days.

Keywords: Alveolar Bone Loss; Antirheumatic Agents; Periodontitis; Phytotherapy; Dental Scaling.

\section{Introduction}

Periodontitis is an immune-inflammatory disease induced by dental biofilm that induces a host response against bacterial antigens, and it is evidenced clinically by the resorption of alveolar bone concomitant with clinical attachment loss and the formation of periodontal pockets. ${ }^{1}$ The traditional treatment for this disease is scaling and root planing (SRP) to remove the bacterial biofilm. ${ }^{2}$ However, despite the good clinical outcomes promoted by SRP in the control of periodontitis, ${ }^{3}$ the application of medications that modulate the host inflammatory response against 
bacterial challenge has been suggested to be able to improve the outcomes of periodontal treatment. ${ }^{4}$ This therapy, termed host modulation therapy, is based on the observation that the progression of periodontal disease is highly dependent on the expression of biological mediators of inflammation and that the alteration of the expression of these proteins modifies the inflammatory profile of the patients and assists in the resolution of this pathological process. ${ }^{5}$

Several immunomodulatory drugs of the inflammatory response have been suggested as alternatives for the adjunctive treatment of periodontitis. ${ }^{4,5}$ Among these drugs, some drugs are noteworthy due to the positive results observed clinically, such as nonsteroidal anti-inflammatory drugs (NSAIDs) selective ${ }^{6}$ or not selective for cyclooxygenase-2 (COX-2), ${ }^{7}$ bisphosphonates, ${ }^{8}$ and low doses of doxycycline. ${ }^{9}$ However, the therapeutic protocol with these drugs has limitations due to the induction of side effects. ${ }^{4}$ Additionally, all of these drugs act only by inhibiting the anti-inflammatory mediators of inflammation, and a good modulating host inflammatory response drug has been suggested to also act to stimulate the biological mediators of tissue repair. ${ }^{4,10}$

Avocado/soybean unsaponifiables (ASU) constitute a medication produced from the fruits and seeds of these two plants in a 1:2 ratio (avocado/soy), and this medication is indicated for the treatment of rheumatoid arthritis and osteoarthritis. ${ }^{11}$ This medication is considered to be a structural modifier of the joint tissues that were previously destroyed by the progression of arthritis/osteoarthritis. ${ }^{12,13,14,15}$ The mechanism of action of this medication may make it applicable as a possible adjunctive agent for periodontal treatment. Clinical studies have found that ASU reduce the intra-articular space and improve joint kinesiology function. ${ }^{2,13,14,15}$ The effects of ASU on the protection and proliferation of intra-articular tissues were verified histologically in studies with sheep ${ }^{14}$ and dogs. ${ }^{13}$ In vitro studies have shown that ASU downregulates the expression of pro-inflammatory mediators, such as PGE2, TNFa, iNOS, IL1 $\beta$, IL6, and IL8, 16,17,18 and matrix metalloproteinases, such as MMP2, MMP3, and MMP13; ${ }^{18,19,20}$ ASU also promotes the upregulation of the expression of growth factors such as TGF $\beta 1$, TGF $\beta 2$, and BMP2 $2^{17,21,22}$ and the synthesis of proteins of the matrix of the connective tissue, such as collagen and agrecans. ${ }^{1718,23}$ Furthermore, in vivo studies have shown that ASU reduces the expression of iNOS and MMP $13^{13}$ and increases the secretion of TGF $\beta 1$ and TGF $\beta 2 .{ }^{24}$

Taking into account the anti-inflammatory and proliferative effects of ASU and the good outcomes induced by this drug in the treatment of arthritis, which possesses a pathogenic mechanism of tissue destruction similar to that which occurs in periodontitis, ${ }^{25}$ this study aimed to assess whether the use of ASU promoted beneficial effects on periodontal repair after the treatment of ligatureinduced periodontitis in rats.

\section{Methodology}

This study was approved by the Ethical Committee for the use of animals in research of the São Paulo State University - UNESP (CEUA 17/2009), School of Dentistry of Araraquara, Brazil. All procedures performed in these studies were in accordance with the ethical standards of this institution. Eighty-four male rats (Rattus norvegicus Holtzman), weighing 250 - $300 \mathrm{~g}$ were kept in an environment with controlled temperature $\left(21 \pm 1^{\circ} \mathrm{C}\right)$, humidity (65-70\%) and light/dark cycle (12 hours). The animals had ad libitum access to a standard laboratory diet and water.

\section{Groups}

The 84 animals were randomly allocated to four equally sized groups in three follow-up periods (follow up at 7, 15, and 30 days after SRP) with seven animals per group/period: a) SRP group - Administration of saline solution (SS); b) SRP/ASU-7 group - Administration of ASU $(0.6 \mathrm{~g} / \mathrm{kg} / \text { day })^{26}$ (Piascledine 300, Expanscience Lab, France) beginning 7 days before the induction of periodontitis; c) SRP/ASU0 group - Administration of ASU beginning on the day of the periodontitis induction; d) SRP/ASU +7 group - Administration of ASU beginning at the moment of the SRP treatment of induced periodontitis. The ASU and SS were 
administered daily by gavage until the end of the follow-up period.

\section{Induction and treatment of periodontitis}

The animals were anaesthetized with a combination of ketamine $(0.08 \mathrm{ml} / 100 \mathrm{~g}$; Rompum, Bayer S.A., São Paulo, Brazil) and xylazine $(0.04 \mathrm{ml} / 100 \mathrm{~g}$; Rompum, Bayer S.A., São Paulo, Brazil). Ligatures (cotton fibres, no. 24) were placed subgingivally around the upper second molar on both sides in five animals per group/period and in one side in two animals per group/period. In these two animals, the side selected for the ligature placement was randomly selected. After a period of 7 days, the ligatures were removed, and scaling and root planning (SRP) was performed once with manual instruments (11-12/ 1314-Mini-Gracey, Hu-Friday, Chicago, USA $)^{27}$ with the aid of a stereoscopic magnifying lens with 3.5X magnification (DMC equipaments, São Carlos, Brazil).

\section{Retrieval of samples}

Euthanasia was performed via an overdose of anaesthetic solution. In each of the groups, the maxillae were removed in blocks and divided randomly into seven hemi-maxillae for histomorphometric/ immunohistochemistry analysis and five hemimaxillae for qPCR and micro-CT analysis; two hemimaxillae without ligatures were used as controls for histology. The hemi-maxillae intended for histomorphometric/immunohistochemistry analysis were fixed in $4 \%$ paraformaldehyde for 48 hours. Hemi-maxillae intended for qPCR had the gingival tissue surrounding the upper second molars removed, immediately frozen in liquid nitrogen and kept at $-80^{\circ} \mathrm{C}$ until the extraction of mRNA; the remaining blocks containing the teeth and surrounding alveolar bone were fixed in $4 \%$ paraformaldehyde for 48 hours and subsequently scanned in a micro-CT scanner.

\section{Micro-CT}

The samples were scanned by a microtomograph (Skyscan, Aartselaar, Belgium) and subsequently reconstructed, spatially reoriented and analysed by specific software (NRecon/DataViewer/CTan, Skyscan, Aartselaar, Belgium). The bone volume in the furcation area in the second molar was measured after the delimitation of the region of interest (ROI), which was an area of $70 \times 45$ pixels $^{2}, 6018-\mu$ m-thick sections, and a threshold range of 55-250 greyscale. The values were given as \% bone tissue in the ROI. A blinded, trained and calibrated examiner (GJO) performed the analyses.

\section{Histology - histomorphometry}

After the fixation, the samples were decalcified in ethylenediamine tetraacetic acid (EDTA) at 7\% for 10 weeks and subsequently dried in alcohol, diaphanized in xylene and embedded in paraffin. Serial sections, $5 \mu \mathrm{m}$ thick, were prepared in a mesiodistal direction along the axis of the tooth and were stained with haematoxylin - eosin (HE). Histological and histomorphometric analysis was performed while viewing the images on a computer screen via a camera connected to an incandescent and polarized light microscope (Leica - Reichert Diastar Products \& Jung, Wetzlar, Germany) and using image analysis software (Image J, Jandel Scientific, San Rafael, USA). After excluding the first and the last sections where the furcation regions were detected, three equidistant sections from each specimen block were selected. The following parameters were evaluated in the histomorphometric analysis: 1) area of mineralized bone within the furcation, expressed as percentage of the furcation area (i.e., from the roof of the furcation to $1000 \mu \mathrm{m}$ apically) ${ }^{28}$ and $\mathrm{b}$ linear distances between the cemento-enamel junction (CEJ) to the top of the alveolar crest (CEJ- CB), at a point where the periodontal ligament showed a normal width, and the CEJ to the apical termination of the junctional epithelium (CEJ- aJE). ${ }^{28}$ Additionally, a histological description of the locations of inflammatory reactions and bone remodelling in the furcation region was performed. Experienced, calibrated, and blinded evaluators (RSN and LGP) performed the histological description and histomorphometric analysis.

\section{Immunohistochemistry}

Sections were prepared according to standard protocols for the identification of tartrate-resistant acid phosphatase (TRAP), the receptor activator of nuclear factor kappa-B ligand (RANKL), and alkaline phosphatase (AP). Immunohistochemical 
staining for TRAP, RANKL and AP was performed using anti-rat antibodies (Abcam, Inc. Cambridge, MA, USA) for these proteins (Dilution: TRAP- 1:200; RANKL-1:200; AP-1:100). Negative control sections were incubated with PBS (omission of the primary antibody) to assess background staining. Biotinylated immunoglobulin (ABC kit Dako A/S, Denmark) was used as a secondary antibody, followed by incubation with the avidin-biotin peroxidase complex (ABC kit Dako, Glostrup, Denmark). Diaminobenzidine (DAB, Dako A/S, Denmark) was used as a chromogenic substrate. All sections were counterstained with Carrazi's haematoxylin and mounted with Permount. Photomicrographs (50X) were taken using a light microscope (LEICA microsystem $\mathrm{GmbH}$, Wetzlar, Germany). The analysis of TRAP was performed by counting the cells in the furcation region and on the top of the crestal bone. ${ }^{28}$ The intensity of AP and RANKL was scored with a 4 -grade scale: (0) no marking (i.e., $0 \%$ of cells); (1) weak ( $0-25 \%$ of cells), (2) moderate (25-50\% of cells), and (3) strong (50-75\% of cells). ${ }^{27}$ These parameters were assessed at the interproximal aspects of the tooth (area delimitated by 1,000 $\mu \mathrm{m}$ below the CEJ between the mesial root of the second molar and the distal root of the first molar and between the distal root of the second molar and the mesial root of the third molar) and within the furcation area (same area of evaluation of the histomorphometry analysis of the percentage of bone in the furcation area), and the median of the values from three regions was used to represent each sample. A blinded, trained and calibrated examiner (GJO) performed the analyses.

\section{Total RNA extraction, cDNA synthesis, and qPCR analysis}

Total RNA was extracted from tissue samples using an affinity column system (RNAqueous-4PCR, Ambion Inc.) according to the manufacturer's protocol. The quantity and purity of total RNA were determined by UV spectrophotometry and the $260 / 280 \mathrm{~nm}$ ratio, respectively. The RNA integrity of a subsample was confirmed by electrophoresis in formaldehyde agarose gels. Approximately $400 \mathrm{ng}$ of total RNA was converted into cDNA with random hexamer primers and moloney leukaemia virus reverse transcriptase in a reaction volume of $20 \mu \mathrm{L}$ (High capacity cDNA synthesis kit, Applied Biosystems).

The qPCR reactions were performed in a $20-\mu \mathrm{L}$ volume reaction including $10 \mu \mathrm{L}$ TaqMan qPCR master mix (Applied Biosystems), $1 \mu \mathrm{L}$ diluted cDNA, $8 \mu \mathrm{L}$ deionized water, and $1 \mu \mathrm{L}$ rat-specific predesigned and optimized pairs of primers and probe. The pre-optimized cycling conditions were as follows: $50^{\circ} \mathrm{C}$ for 2 minutes, $95^{\circ} \mathrm{C}$ for 10 minutes and 40 cycles of $95^{\circ} \mathrm{C}$ for 15 seconds and $60^{\circ} \mathrm{C}$ for 1 minute. The qPCR was performed in a Real-Time PCR System 7500 (Applied Biosystems). The relative levels of gene expression for IL-1 $\beta$, IL-6, RANKL, TNF- $\alpha$, and AP were calculated using GAPDH as a normalizing gene.

\section{Statistics}

A statistical package (GraphPad Prism 5, San Diego, USA) was used to perform all analyses. The sample size calculation was based on our previous study, which evaluated the effect of ASU on the periodontal repair of induced periodontitis in rats without performing SRP, with similar histomorphometric methodology. ${ }^{28}$ Thus, with seven animals in each group, a minimum average difference between groups of $26.20 \%$ with a standard deviation of $12.91 \%$ could be detected with a power of $80 \%$ and an alpha error of 0.05 .

The reproducibility of the examiners' micro-CT, histomorphometry (\% bone fill), and immunohistochemistry analyses was analysed from duplicate measurements of 10 samples with a 1-week interval. No differences between the evaluations were shown with the paired t-test and Wilcoxon test $(p>0.05)$. In addition, Pearson's correlation showed an intra-examiner correlation of $r=0.94$ for micro-CT, $\mathrm{r}=0.85$ for histomorphometry, and $\mathrm{r}=0.91$ for TRAP. Spearman's correlation showed an intra-examiner correlation of $\mathrm{r}=0.92$ for $\mathrm{AP}$ and $\mathrm{r}=0.94$ for RANKL.

The normality of the continuous variables was confirmed with the Shapiro-Wilk test ( $p>0.05)$, and differences among groups were evaluated with ANOVA and Tukey's post hoc test. Analysis of the categorical variables was performed with 
the Kruskal-Wallis test and Dunns' test. The level of significance was set at $\mathrm{p} \leq 0.05$.

\section{Results}

\section{Micro-CT}

The micro-CT analysis showed no statistically significant differences between groups in any of the periods. Additionally, no differences were found within each group. Table 1 shows the averages and standard deviations of the volumetric analysis of $\%$ bone tissue.

\section{Descriptive Histology}

The control specimens of all of the groups presented an organized connective tissue without a huge inflammatory infiltrate and bone with normal shape in all of the periods. Regarding the histological aspects of the teeth where the periodontitis was induced, all of the groups presented a similar histological pattern in which the presence of inflammatory infiltrate in the roof of the furcation, the presence of blood vessels associated with this infiltrate and surrounding bone tissue, and disorganization of collagen fibres were found at 7 days. At 15 and 30 days, a reduction in the inflammatory infiltrate, which was replaced by organized collagen fibres, associated with a reduction in the presence of blood vessels and improved bone tissue organization was observed. Furthermore, the presence of reversal lines demonstrated bone formation in all groups. Representative images of the descriptive histological analysis are shown in Figure 1.

\section{Histomorphometry}

The SRP/ASU+7 group was shown to present a higher \% bone in the furcation area than in the SRP group after a period of 7 days $(\mathrm{p}<0.05)$. An increase in the \% bone in the furcation region in the SRP group at 30 days relative to that at 7 days $(p<0.05)$ was also shown in the intragroup comparison. Table 2 shows the averages and standard deviations of the \% bone in the furcation regions. Regarding the analysis of the CEJ-CB distance, there were no differences between the groups in all of the periods evaluated. In the intragroup analysis, a reduction in the CEJ-CB in the SRP/ASU-7 group
Table 1. Average and standard deviation of the volumetric analysis of the percentage of bone detected by micro-CT (\%) in all of the groups/periods.

\begin{tabular}{lccc}
\hline Group/Period & 7 days & 15 days & 30 days \\
\hline SRP & $19.71 \pm 6.82$ & $22.22 \pm 9.34$ & $21.15 \pm 4.30$ \\
SRP+ASU/-7 & $18.48 \pm 6.16$ & $21.58 \pm 5.28$ & $19.40 \pm 6.37$ \\
SRP+ASU/0 & $16.73 \pm 3.70$ & $18.11 \pm 5.24$ & $17.14 \pm 7.22$ \\
SRP+ASU/+7 & $17.32 \pm 3.70$ & $18.31 \pm 4.65$ & $15.02 \pm 6.70$
\end{tabular}

SRP: scaling and root planning; ASU: avocado/soybean unsaponifiables.

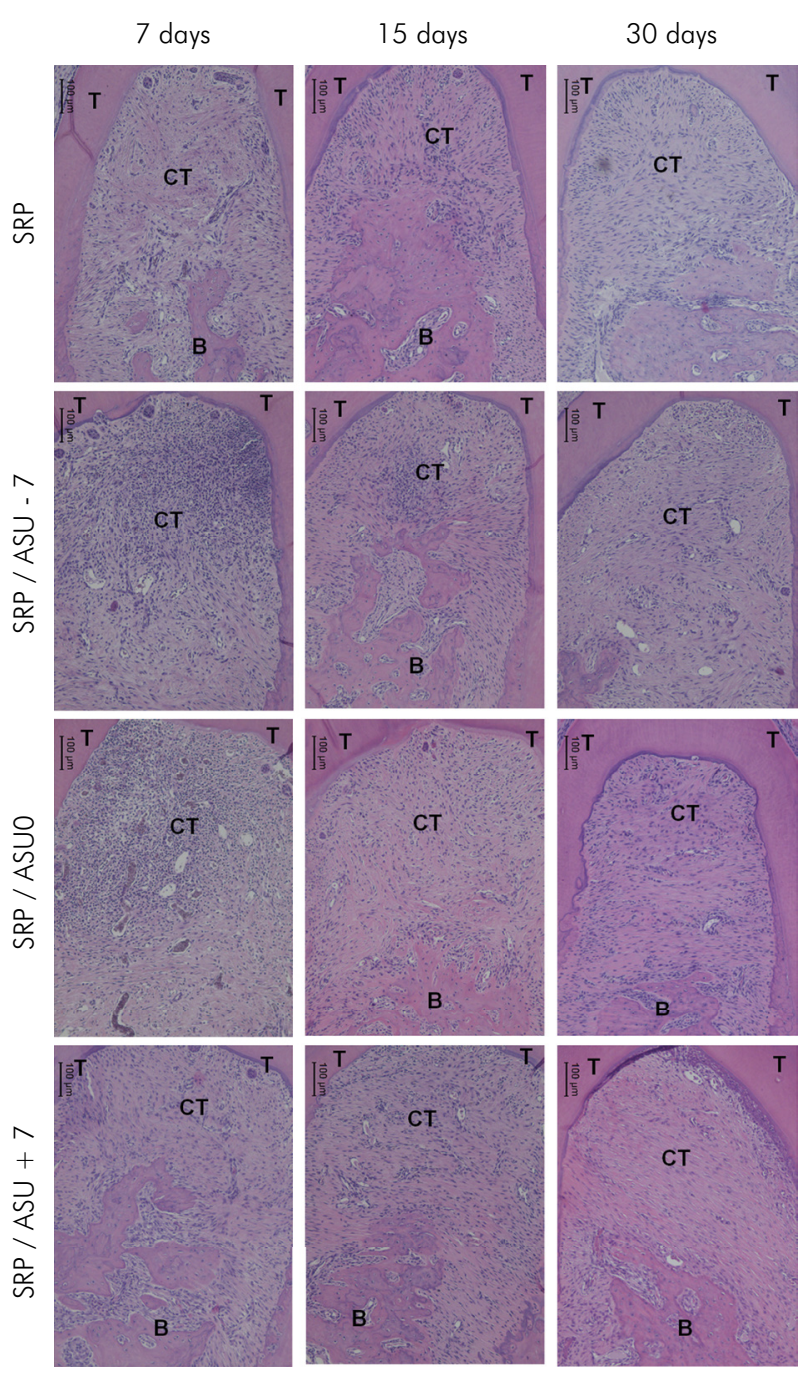

Figure 1. Representative images of the histological analysis in all of the groups/periods of evaluation. The healing pattern was similar in all groups, where a reduction in the inflammatory infiltrate, an increase in the organization of the connective tissue and the formation of osteoid tissue were observed in all of the groups with increased evaluation periods. B-Bone; T-Teeth; CT-Connective tissue, iCT-Inflammatory infiltration in the connective tissue. (100X) HE. 
Table 2. Average and standard deviation of the percentage of bone fill in the furcation (\%) in all of the groups/periods.

\begin{tabular}{lccc}
\hline Group/Period & 7 days & 15 days & 30 days \\
\hline SRP/SS & $9.90 \pm 10.65^{a, b}$ & $17.57 \pm 10.68$ & $35.05 \pm 18.48^{b}$ \\
SRP+ASU/-7 & $20.30 \pm 15.51$ & $24.34 \pm 18.75$ & $33.38 \pm 21.08$ \\
SRP+ASU/0 & $24.27 \pm 14.78$ & $26.60 \pm 17.89$ & $32.71 \pm 16.76$ \\
& & \\
SRP+ASU/+7 & $29.84 \pm 16.34^{a}$ & $25.12 \pm 12.07$ & $38.25 \pm 19.15$ \\
\hline $\begin{array}{l}\text { SRP: scaling and root planning; SS: saline solution; ASU: avocado/ } \\
\text { soybean unsaponifiables. }{ }^{\circ} \mathrm{p}<0.05-\text { Intergroup differences, } \\
\text { One-way ANOVA and Tukey's post test; }{ }^{b} p<0.05 \text {-Intragroup } \\
\text { differences, One-way ANOVA and Tukey's post test }\end{array}$
\end{tabular}

Table 3. Average and standard deviation of the CEJ-CB (mm) in all of the groups/periods.

\begin{tabular}{|c|c|c|c|}
\hline Groups/Periods & 7 days & 15 days & 30 days \\
\hline SRP/SS & $1.59 \pm 0.27$ & $1.53 \pm 0.15$ & $1.30 \pm 0.17$ \\
\hline $\mathrm{SRP}+\mathrm{ASU} /-7$ & $1.27 \pm 0.18^{a}$ & $1.54 \pm 0.31^{b}$ & $1.04 \pm 0.14^{a}$ \\
\hline $\mathrm{SRP}+\mathrm{ASU} / 0$ & $1.33 \pm 0.29$ & $1.48 \pm 0.29^{b}$ & $1.15 \pm 0.18^{b}$ \\
\hline $\mathrm{SRP}+\mathrm{ASU} /+7$ & $1.38 \pm 0.28$ & $1.49 \pm 0.31$ & $1.29 \pm 0.27$ \\
\hline \multicolumn{4}{|c|}{$\begin{array}{l}\text { SRP: scaling and root planning; SS: saline solution; ASU: avocado/ } \\
\text { soybean unsaponifiables. }{ }^{\circ} p<0.05 \text {-Intragroup differences between } \\
\text { the periods of } 7 \text { and } 30 \text { days, One-way ANOVA and Tukey's post } \\
\text { test; }{ }^{b} p<0.05 \text {-Intragroup differences between the periods of } 15 \\
\text { and } 30 \text { days, One-way ANOVA and Tukey's post test }\end{array}$} \\
\hline
\end{tabular}

Table 4. Average and standard deviation of the CEJ-aJE (mm) in all of the groups/periods.

\begin{tabular}{cccc}
\hline Groups/Periods & 7 days & 15 days & 30 days \\
\hline SRP/SS & $0.81 \pm 0.36$ & $0.87 \pm 0.22$ & $0.61 \pm 0.10$ \\
SRP+ASU/-7 & $0.57 \pm 0.17$ & $0.83 \pm 0.30^{\circ}$ & $0.64 \pm 0.12^{\circ}$ \\
SRP +ASU/0 & $0.59 \pm 0.20$ & $0.75 \pm 0.33$ & $0.55 \pm 0.15$ \\
& & & \\
SRP+ASU/+7 & $0.49 \pm 0.20$ & $0.72 \pm 0.18$ & $0.68 \pm 0.28$ \\
$\begin{array}{l}\text { SRP: scaling and root planning; SS: saline solution; ASU: avocado/ } \\
\text { soybean unsaponifiables }{ }^{\circ} \mathrm{p}<0.05 \text {-Intragroup differences between the } \\
\text { periods of } 15 \text { and } 30 \text { days, One-way ANOVA and Tukey's post test }\end{array}$
\end{tabular}

at the period of 30 days was shown compared with that at 7 and 15 days $(p<0.05)$. Furthermore, in the SRP/ASU0 group, a reduction in the CEJ-CB was shown at 30 days compared with that at 15 days. Table 3 shows the average and standard deviation of the CEJ-CB distance.

Regarding the CEJ-aJE distance, there were no differences between the groups in any of the follow-up periods. In the intragroup analysis, a reduction in the CEJ-aJE distance at 30 days compared with that at 15 days was shown in the SRP/ASU-7 group $(p<0.05)$. Table 4 shows the average and standard deviation of the CEJ-aJE distance.

\section{Immunohistochemistry}

No statistically significant differences were detected between groups in any of the proteins analysed. Figure 2 shows representative images of all groups with respect to the immunohistochemical labelling of the proteins evaluated.

\section{qPCR Analysis}

No differences in the expression levels of IL1 $\beta$, IL6, or TNFa mRNA between the groups at any period of evaluation were observed. RANKL mRNA was expressed at higher levels in the SRP and SRP/ASU-7 groups than in the SRP/ASU0 and SRP/ASU+7 groups at 15 days $(\mathrm{p}<0.05)$. AP mRNA was expressed at higher levels in the SRP/ASU+7 group than in all of the other groups at 30 days ( $p<0.001)$. Figure 3 shows a graphic representation of the averages and standard deviations of the mRNA expression of AP, RANKL, IL1 $\beta$, IL6, and TNFa in relation to GAPDH gene expression.

\section{Discussion}

The results of this study showed that in general, the adjunctive use of ASU exerts a subtle additional effect on periodontal repair after the treatment of induced periodontitis, as demonstrated by histologic, histomorphometric and micro-CT analysis. Furthermore, despite the higher expression of AP in the $\mathrm{SRP} / \mathrm{ASU}+7$ group than in all of the other groups and a lower expression of RANKL in the SRP/ASU0 and SRP/ASU+7 groups than in the SRP and SRP/ASU-7 

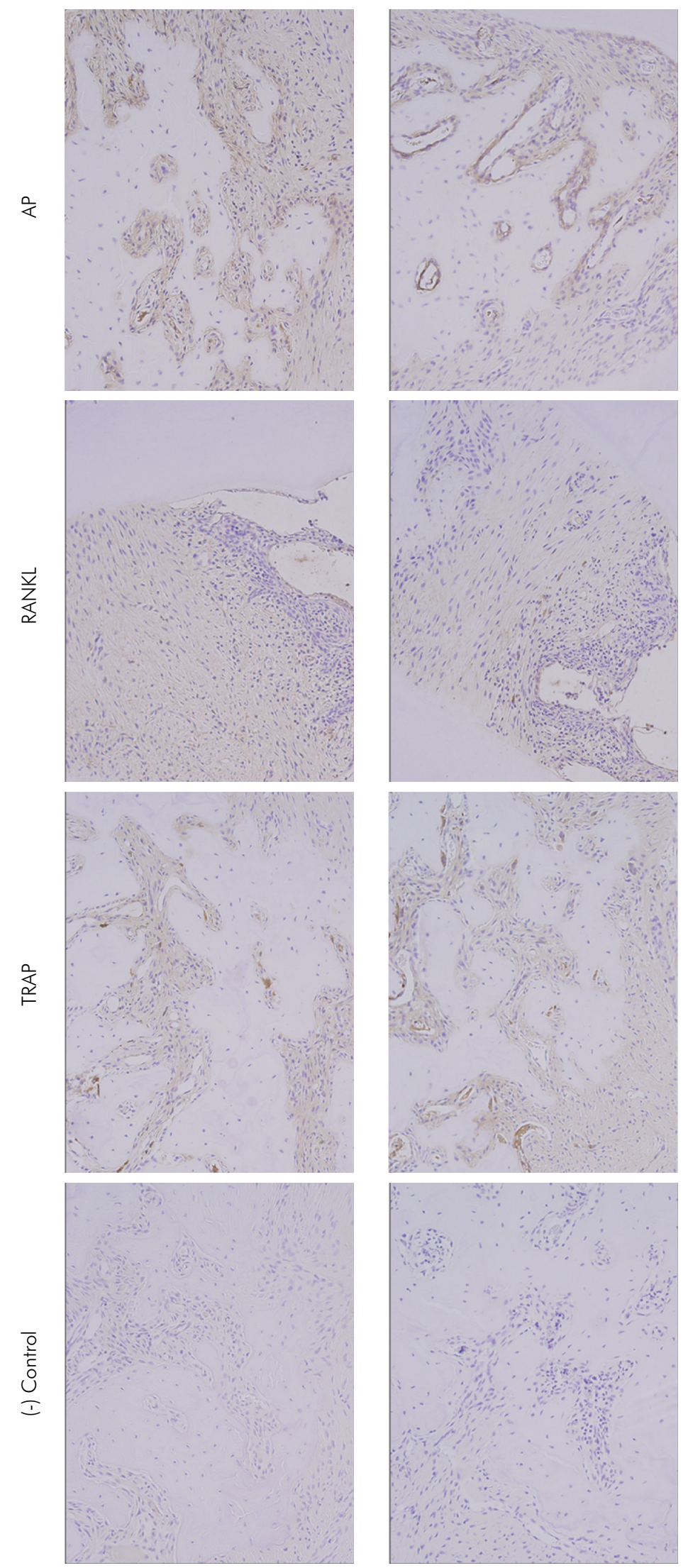

dyS
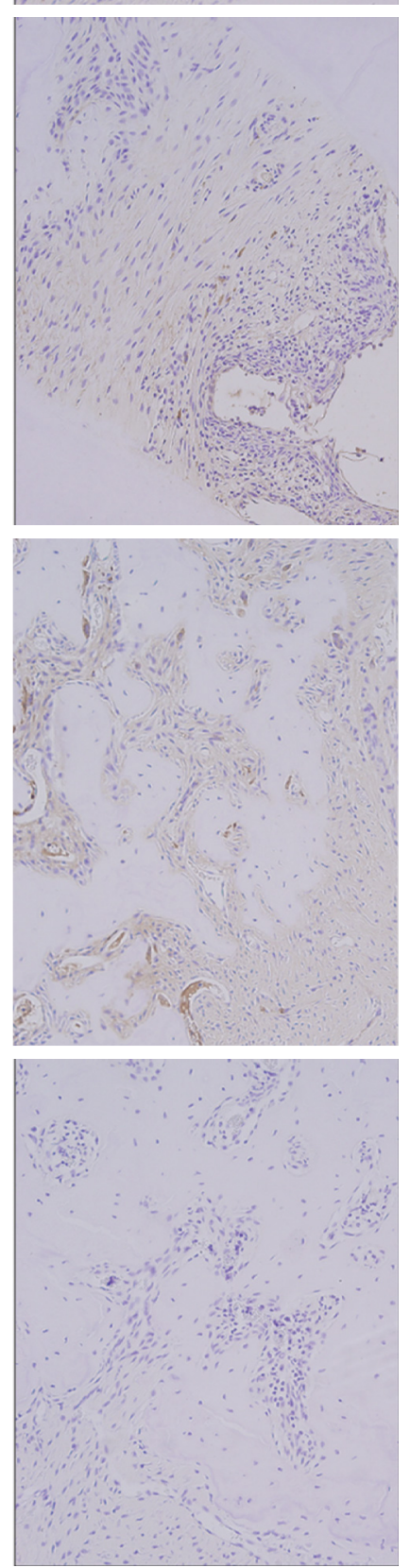

กS $\forall$





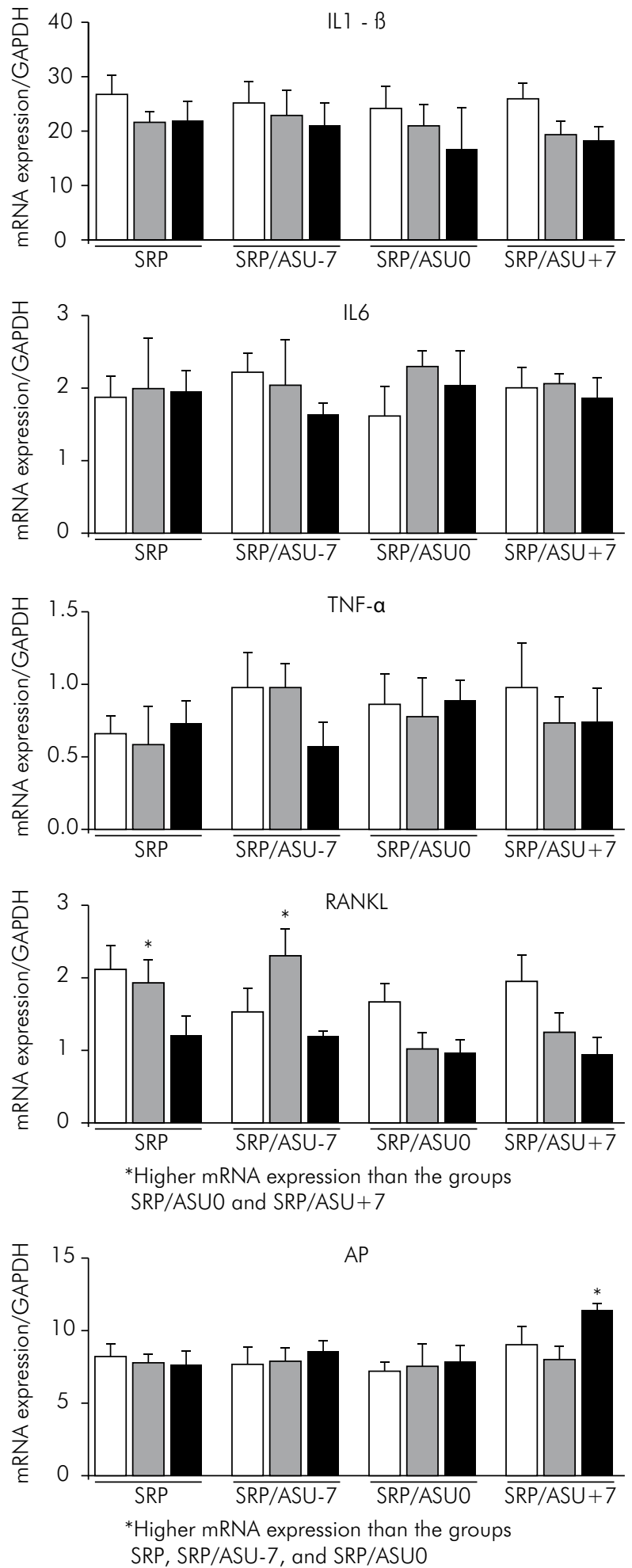

7 days

15 days

30 days

Figure 3. Representative graphs of the average, standard deviation, and significant differences among the groups in the expression of mRNA for IL1 $\beta$, IL6, TNF, RANKL, and AP. The data were normalized with the housekeeping gene (GAPDH). groups, these differences in gene expression were not detected by immunohistochemical analysis.

At the 7-day follow-up, the animals of the SRP/ASU+7 group presented a higher percentage of bone fill in the furcation regions than the SRP group. It can be assumed that this effect must be due to anti-inflammatory and proliferative effects in the connective tissue that were induced by the ASU treatment. ${ }^{13,14,15,17,20,21}$ However, these results were not maintained in the other periods or confirmed by the other analyses, such as the CEJ-CB and the volumetric \% bone analysis performed by the micro-CT. The discrepancy between the data obtained from the analysis of the \% bone in the furcation with the data of the distance between the CEJ-CB and CEJ-aJE at 7 days might be due to the different areas of assessment; while the first analysis was performed at the furcation, the other analyses were performed in the interproximal region. The discrepancy between the results of the histomorphometric and micro-CT analyses in the furcation regions might be due to the extension of the analysis, because only three sections in two-dimensional images were evaluated histomorphometry, while micro-CT performed a tridimensional analysis of the entire furcation region. ${ }^{29}$

Comparing the histomorphometric and the micro-CT data of this study with those of a previous study performed by our research group, distinct results were detected..$^{28}$ The treatment with ASU beginning at the day of the ligature removal induced a lower degree of bone resorption, a lower number of osteoclasts, lower expression of RANKL and IL1 $\beta$, and a higher expression of AP than in the control group ${ }^{28}$ but these results were not confirmed by this study. The different models for the evaluation of the periodontal repair after induced periodontitis in rats could explain the distinct results presented in these studies. In the first study, the model of periodontal repair consisted of the removal of the ligatures 7 days after their insertion, ${ }^{28}$ while in this study, SRP was performed with manual instruments at the time of ligature removal. ${ }^{27,30}$ Despite the fact that the depths of the periodontal pockets were not measured in this study, attachment loss verified by the linear measures of the CEL-CB distance was shown to vary 
in the range of $1.27-1.59 \mathrm{~mm}$, and it can be assumed that the depth of the periodontal pockets formed by the induction of periodontitis was less than $1.5 \mathrm{~mm}$. Although the manual instruments used to perform the SRP had a tip with a reduced size (mini-five), the active tip of this curette was relatively large in relation to the periodontal pockets that were present in the animals of the present study. The trauma caused by SRP in shallow periodontal pockets has been shown to induce clinical attachment loss in humans. ${ }^{31,32}$ The additional trauma in the dentogingival insertion caused by SRP may have impaired the possible positive effects of ASU treatment in periodontal repair. Another explanation for these distinct results is that the additional removal of the bacteria debris by SRP in this model may improve the outcomes in the control group. It is possible that the adjunctive therapy with ASU only improved the treatment of deep periodontal pockets where SRP presented poor clinical outcomes. ${ }^{3}$

It was shown in this study that the SRP/ASU0 and SRP/ASU+7 groups presented lower expression of RANKL mRNA than the SRP and SRP/ASU-7 groups at 15 days. Additionally, it was shown that the SRP/ASU+7 group presented a higher expression of AP mRNA than all of the other groups at 30 days. These results indicated that ASU treatment produced subtle anti-inflammatory and proliferative effects but did not elicit changes in protein synthesis or periodontal repair. ASU is considered to be similar to medications that modify the structure of connective tissue; however, these effects occur at a slow rate, ${ }^{12,13}$ and the increase in the expression of AP mRNA at 30 days could represent a possible enhancement of the periodontal repair in longer periods of evaluation, though this fact cannot be proven by this study.

When evaluating the results presented by this study, some clear drawbacks should be considered. One limitation of this study was that only one concentration of ASU was tested, and despite the fact that clinical and pre-clinical studies have shown that an increase in the dosage did not produce different clinical or histological effects in rheumatoid arthritis and osteoarthritis, ${ }^{12,14}$ in vitro studies have shown that ASU produces effects in a dose-dependent manner. ${ }^{16,20}$ Therefore, the impact of different dosages of ASU on periodontal repair remains unknown. Another limitation of this study is the fact that ASU is a slow-acting drug, and this may mean that the effect of this drug will be better detected in longer evaluation periods. Indeed, previous preclinical studies from our research group detected the acceleration of the osseointegration of implants inserted in the tibia and greater bone formation in critically sized calvaria defects after using ASU for periods between $60-75$ days $^{33}$, and this effect was associated with increased expression of the growth factors BMP2 and TGF $\beta 1 .{ }^{33}$ Furthermore, due to the inconsistent effects of ASU in the different models of the evaluation of the periodontal repair after inducing periodontitis with ligatures, additional studies on the effect of ASU in other models of induced periodontitis (e.g., maintenance of the ligature in the position or the injection of lipopolysaccharide) and studies with animals with conditions that predispose them to higher bone resorption (e.g., diabetes, smoking) will be necessary to elicit a better understanding of the real effect of ASU on induced periodontitis and in which situations this drug may produce more significant effects.

\section{Conclusion}

Taking into account the results and methodology applied, it can be concluded that the use of ASU on the day of the SRP treatment of the ligature-induced periodontis promotes subtle beneficial effects on periodontal repair following the treatment of induced periodontitis within the experimental period of 7 days.

\section{Acknowledgements}

This study was financed by the Brazilian agency Fapesp (Fundação de amparo a pesquisa do estado de São Paulo (2010/13029-9 and 2009/17395-2). The authors declare no other conflict of interest regarding this study. The authors would like to thank the laboratory technicians Leandro Alves dos Santos and Ana Claudia Gregolin Costa Miranda for their support. 


\section{References}

1. Van Dyke TE, Serhan CN. Resolution of inflammation: a new paradigm for the pathogenesis of periodontal diseases. J Dent Res. 2003;82(2):82-90. https://doi.org/10.1177/154405910308200202

2. Heitz-Mayfield LJ, Lang NP. Surgical and nonsurgical periodontal therapy. Learned and unlearned concepts. Periodontol 2000. 2013;62(1):218-31. https://doi.org/10.1111/prd.12008

3. Lindhe J, Westfelt E, Nyman S, Socransky SS, Haffajee AD. Long-term effect of surgical/non-surgical treatment of periodontal disease. J Clin Periodontol. 1984;11(7):448-58. https://doi.org/10.1111/j.1600-051X.1984.tb01344.x

4. Kantarci A, Hasturk H, Van Dyke TE. Hostmediated resolution of inflammation in periodontal diseases. Periodontol 2000. 2006;40(1):144-63. https://doi.org/10.1111/j.1600-0757.2005.00145.x

5. Kirkwood KL, Cirelli JA, Rogers JE, Giannobile WV. Novel host response therapeutic approaches to treat periodontal diseases. Periodontol 2000. 2007;43:294-315. https://doi.org/10.1111/j.1600-0757.2006.00166.x

6. Yen CA, Damoulis PD, Stark PC, Hibberd PL, Singh M, Papas AS. The effect of a selective cyclooxygenase-2 inhibitor (celecoxib) on chronic periodontitis. J Periodontol. 2008;79(1):104-13. https://doi.org/10.1902/jop.2008.070271

7. Williams RC, Jeffcoat MK, Howell TH, Rolla A, Stubbs D, Teoh KW et al. Altering the progression of human alveolar bone loss with the non-steroidal anti-inflammatory drug flurbiprofen. J Periodontol. 1989;60(9):485-90. https://doi.org/10.1902/jop.1989.60.9.485

8. Lane N, Armitage GC, Loomer P, Hsieh S, Majumdar $\mathrm{S}$, Wang HY et al. Bisphosphonate therapy improves the outcome of conventional periodontal treatment: results of a 12-month, randomized, placebocontrolled study. J Periodontol. 2005;76(7):1113-22. https://doi.org/10.1902/jop.2005.76.7.1113

9. Emingil G, Atilla G, Sorsa T, Luoto H, Kirilmaz L, Baylas $\mathrm{H}$. The effect of adjunctive low-dose doxycycline therapy on clinical parameters and gingival crevicular fluid matrix metalloproteinase-8 levels in chronic periodontitis. J Periodontol. 2004;75(1):106-15. https://doi.org/10.1902/jop.2004.75.1.106

10. Bhatavadekar NB, Williams RC. New directions in host modulation for the management of periodontal disease. J Clin Periodontol. 2009;36(2):124-6. https://doi.org/10.1111/j.1600-051X.2008.01354.x

11. Cameron M, Gagnier JJ, Chrubasik S. Herbal therapy for treating rheumatoid arthritis. Cochrane Database Syst Rev. 2011;(2):CD002948. https://doi.org/10.1002/14651858.CD002948.pub2

12. Appelboom T, Schuermans J, Verbruggen G, Henrotin Y, Reginster JY. Symptoms modifying effect of avocado/soybean unsaponifiables (ASU) in knee osteoarthritis. A double blind, prospective, placebocontrolled study. Scand J Rheumatol. 2001;30(4):242-7. https://doi.org/10.1080/030097401316909602

13. Boileau C, Martel-Pelletier J, Caron J, Msika P, Guillou GB, Baudouin C et al. Protective effects of total fraction of avocado/soybean unsaponifiables on the structural changes in experimental dog osteoarthritis: inhibition of nitric oxide synthase and matrix metalloproteinase-13. Arthritis Res Ther. 2009;11(2):R41. https://doi.org/10.1186/ar2649

14. Cake MA, Read RA, Guillou B, Ghosh P. Modification of articular cartilage and subchondral bone pathology in an ovine meniscectomy model of osteoarthritis by avocado and soya unsaponifiables (ASU). Osteoarthritis Cartilage. 2000;8(6):404-11. https://doi.org/10.1053/joca.1999.0315

15. Maheu E, Cadet C, Marty M, Moyse D, Kerloch I, Coste P et al. Randomised, controlled trial of avocado-soybean unsaponifiable (Piascledine) effect on structure modification in hip osteoarthritis: the ERADIAS study. Ann Rheum Dis. 2014;73(2):376-84. https://doi.org/10.1136/annrheumdis-2012-202485

16. Au RY, Al-Talib TK, Au AY, Phan PV, Frondoza CG. Avocado soybean unsaponifiables (ASU) suppress TNF-alpha, IL-1beta, COX-2, iNOS gene expression, and prostaglandin E2 and nitric oxide production in articular chondrocytes and monocyte/macrophages. Osteoarthritis Cartilage. 2007;15(11):1249-55. https://doi.org/10.1016/j.joca.2007.07.009

17. Henrotin YE, Deberg MA, Crielaard JM, Piccardi N, Msika P, Sanchez C. Avocado/soybean unsaponifiables prevent the inhibitory effect of osteoarthritic subchondral osteoblasts on aggrecan and type II collagen synthesis by chondrocytes. J Rheumatol. 2006;33(8):1668-78.

18. Henrotin YE, Sanchez C, Deberg MA, Piccardi N, Guillou GB, Msika P et al. Avocado/soybean unsaponifiables increase aggrecan synthesis and reduce catabolic and proinflammatory mediator production by human osteoarthritic chondrocytes. J Rheumatol. 2003;30(8):1825-34.

19. Gabay O, Gosset M, Levy A, Salvat C, Sanchez C, Pigenet $A$ et al. Stress-induced signaling pathways in hyalin chondrocytes: inhibition by Avocado-Soybean Unsaponifiables (ASU). Osteoarthritis Cartilage. 2008;16(3):373-84. https://doi.org/10.1016/j.joca.2007.06.016

20. Kut-Lasserre C, Miller CC, Ejeil AL, Gogly B, Dridi $\mathrm{M}$, Piccardi $\mathrm{N}$ et al. Effect of avocado and soybean unsaponifiables on gelatinase A (MMP-2), stromelysin 1 (MMP-3), and tissue inhibitors of matrix metalloproteinase (TIMP- 1 and TIMP-2) secretion by human fibroblasts in culture. J Periodontol. 2001;72(12):1685-94. https://doi.org/10.1902/jop.2001.72.12.1685 
21. Andriamanalijaona $\mathrm{R}$, Benateau $\mathrm{H}$, Barre PE, Boumediene $\mathrm{K}$, Labbe D, Compere JF et al. Effect of interleukin-1beta on transforming growth factor-beta and bone morphogenetic protein-2 expression in human periodontal ligament and alveolar bone cells in culture: modulation by avocado and soybean unsaponifiables. J Periodontol. 2006 Jul;77(7):115666. https://doi.org/10.1902/jop.2006.050356

22. Boumediene K, Felisaz N, Bogdanowicz P, Galera P, Guillou GB, Pujol JP. Avocado/soya unsaponifiables enhance the expression of transforming growth factor beta1 and beta 2 in cultured articular chondrocytes. Arthritis Rheum. 1999 Jan;42(1):148-56. https://doi.org/10.1002/15290131(199901)42:1<148::AID-ANR18>3.0.CO;2-U

23. Lippiello L, Nardo JV, Harlan R, Chiou T. Metabolic effects of avocado/soy unsaponifiables on articular chondrocytes. Evid Based Complement Alternat Med. 2008 Jun;5(2):191-7. https://doi.org/10.1093/ecam/nem132

24. Altinel L, Saritas ZK, Kose KC, Pamuk K, Aksoy Y, Serteser M. Treatment with unsaponifiable extracts of avocado and soybean increases TGF-beta1 and TGFbeta2 levels in canine joint fluid. Tohoku J Exp Med. 2007;211(2):181-6. https://doi.org/10.1620/tjem.211.181

25. Berthelot JM, Le Goff B. Rheumatoid arthritis and periodontal disease. Joint Bone Spine. 2010;77(6):537-41. https://doi.org/10.1016/j.jbspin.2010.04.015

26. Yaman M, Eser O, Cosar M, Bas O, Sahin O, Mollaoglu $\mathrm{H}$ et al. Oral administration of avocado soybean unsaponifiables (ASU) reduces ischemic damage in the rat hippocampus. Arch Med Res. 2007;38(5):489-94. https://doi.org/10.1016/j.arcmed.2007.01.008

27. Garcia VG, Gualberto Júnior EC, Fernandes LA, Bosco AF, Hitomi Nagata MJ, Casatti CA et al. Adjunctive antimicrobial photodynamic treatment of experimentally induced periodontitis in rats with ovariectomy. J Periodontol. 2013;84(4):556-65. https://doi.org/10.1902/jop.2012.120163

28. Oliveira GJ, Paula LG, Souza JA, Spin-Neto R, Stavropoulos A, Marcantonio RA. Effect of avocado/soybean unsaponifiables on ligature-induced bone loss and bone repair after ligature removal in rats. J Periodontal Res. 2016;51(3):332-41. https://doi.org/10.1111/jre.12312

29. Chappard D, Retailleau-Gaborit N, Legrand E, Baslé MF, Audran M. Comparison insight bone measurements by histomorphometry and microCT. J Bone Miner Res. 2005;20(7):1177-84. https://doi.org/10.1359/JBMR.050205

30. Garcia VG, Takano RY, Fernandes LA, de Almeida JM, Theodoro LH. Treatment of experimental periodontal disease by a selective inhibitor of cyclooxygenase- 2 with scaling and root planing (SRP). Inflammopharmacology. 2010;18(6):293-301. https://doi.org/10.1007/s10787-010-0059-Z

31. Alves RV, Machion L, Casati MZ, Nociti Júnior FH, Sallum AW, Sallum EA. Attachment loss after scaling and root planing with different instruments. A clinical study. J Clin Periodontol. 2004;31(1):12-5. https://doi.org/10.1111/j.0303-6979.2004.00433.x

32. Heitz-Mayfield LJ, Trombelli L, Heitz F, Needleman I, Moles D. A systematic review of the effect of surgical debridement vs non-surgical debridement for the treatment of chronic periodontitis. J Clin Periodontol. 2002;29(Suppl 3):92-102. https://doi.org/10.1034/j.1600-051X.29.s3.5.x

33. Oliveira GJ, Paula LG, Spin-Neto R, Stavropoulos A, Spolidório LC, Marcantonio E Jr et al. Effect of avocado/ soybean unsaponifiables on osseointegration: a proof-ofprinciple preclinical in vivo study. Int J Oral Maxillofac Implants. 2014;29(4):949-57. https://doi.org/10.11607/jomi.3498 